\title{
Treatment options in fibromuscular dysplasia of the renal artery: when percutaneous transluminal angioplasty is at high risk?
}

Hypertension Research (2013) 36, 383-384; doi:10.1038/hr.2012.193; published online 10 January 2013

\section{CASE REPORT}

A 26-year-old woman was seen in the emergency room for recent onset hypertension. She reported no relevant systemic symptoms, clinical evaluation was unremarkable and blood pressure was 250/150 mm Hg. Blood tests revealed only mild hypokalemia $\left(3.4 \mathrm{mEql}^{-1}\right)$. Target organ damage was documented (microalbuminuria, grade 2 hypertensive retinopathy and left ventricular hypertrophy with diastolic dysfunction on echocardiography). Basal and captopril stimulated plasma renin activity and aldosterone concentration were within the normal limits.

C-reactive protein, erythrocyte sedimentation rate, antinuclear antibodies, a-neutrophil cytoplasmic antibody and antiphospholipid antibodies panel were negative. On ultrasound examination, the right kidney size was decreased with a slight increase of Doppler flow velocity on the right renal artery. Sequential renal scintigraphy showed right kidney hypoperfusion: the right kidney glomerular filtration rate was $37 \mathrm{ml} \mathrm{min}^{-1}$ with patchy hypoperfusion of the upper pole, while the left kidney glomerular filtration rate was $67 \mathrm{ml} \mathrm{min}^{-1}$. Abdominal CT and magnetic resonance angiography (MRA) showed reduced calibre of the right renal artery in its medial and distal tract. The patient was treated with ACE inhibitor and calciumchannel blocker with a transient blood pressure reduction (down to $140 / 94 \mathrm{~mm} \mathrm{Hg}$, mean of repeated office measurements).

Percutaneous transluminal balloon angioplasty (PTA) was planned. On selective renal angiography, 95\% annular stenosis of the main right renal artery at kidney hilum was confirmed (Figure 1). As stenosis was close to the artery bifurcation, PTA-induced artery dissection may have further compromised renal perfusion requiring emergency revascularization or, more likely, nephrectomy. Therefore, the patient was referred to elective vascular surgery. A right renal artery bypass was done with right termino-lateral aorto-renal bypass with the great saphenous vein. The bypass was anastomosed with the right renal artery at the prehilar bifurcation (Figure 2). Revascularization was accompanied by immediate and medium-term significant blood pressure reduction without antihypertensive treatment: mean home blood pressure was $130 / 85 \mathrm{~mm}$ $\mathrm{Hg}$, ambulatory blood pressure was 117/ $80 \mathrm{~mm} \mathrm{Hg}$ at daytime, $111 / 75 \mathrm{~mm} \mathrm{Hg}$ at night-time 6 months after surgery. At 2 years after revascularization, the patient is well, under ramipril $2.5 \mathrm{mg}$ once daily with good
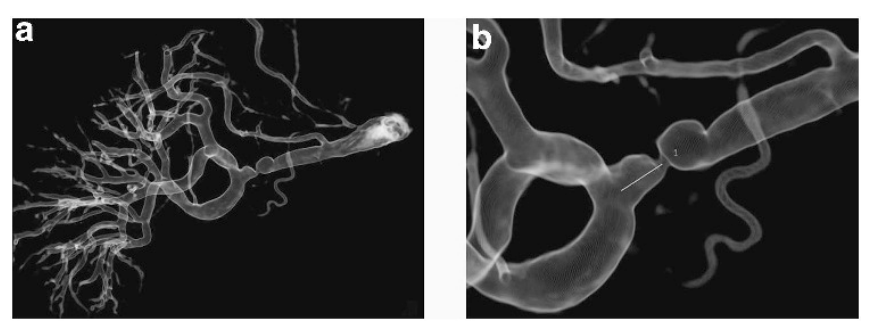

Figure 1 Rotational angiography volume-rendering technique reconstruction showing right renal vascularisation (a) and reduced calibre of the right renal artery in its medial and distal tract (b). office blood pressure control $(138 / 76 \mathrm{~mm} \mathrm{Hg})$, normal urinalysis and renal function (serum creatinine $0.83 \mathrm{mg} \mathrm{dl}^{-1}$, estimated CockroftGault glomerular filtration rate $130 \mathrm{ml} \mathrm{min}^{-1}$ ) and bypass patency (Figure 3).

\section{DISCUSSION}

Fibromuscular dysplasia (FMD) is a nonatherosclerotic non-inflammatory vascular disease that affects young and middle-aged women. It may involve the renal and carotid arteries and it is often underdiagnosed because of the lack of specific symptoms and signs. FMD is classified into three categories according to the affected layer of the arterial wall-intima, media and adventitia (periarterial). Medial FMD is the most common and is further subdivided into medial and perimedial fibroplasia, and medial hyperplasia.

Several diagnostic options can be chosen to detect FMD. MRA can be successfully used for diagnosis, but CT angiography holds the greatest accuracy with sensitivity and specificity reported as $64-99 \%$ and $89-98 \%$, respectively. Catheter-based angiography remains the most accurate imaging technique, but it is performed only when interventional revascularization is planned, as was the case in our patient. ${ }^{1}$

The primary goal in the treatment of patients with renal artery FMD is hypertension control to prevent target organ damage. In patients with non-atherosclerotic renal artery disease associated with severe hypertension, endovascular or open renal revascularization is superior to medical management alone. However, PTA, with or without stenting, and surgical intervention are offered only in case of poor blood pressure control, intolerable side effects of the 


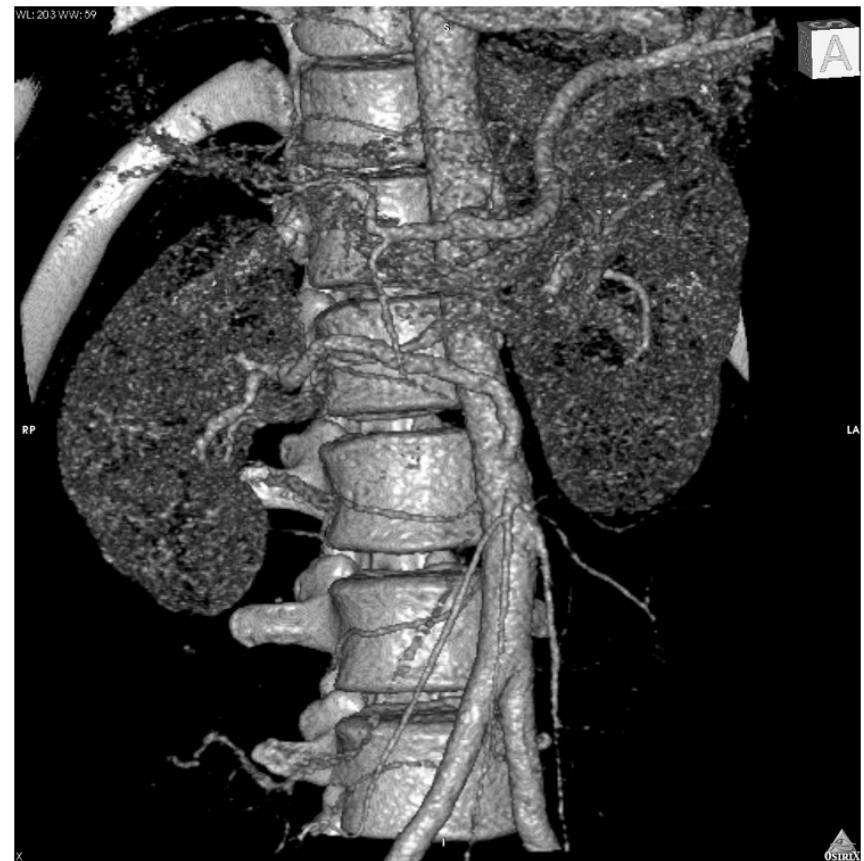

Figure 2 Postoperative angioCT showing venous bypass anastomosed with the right renal artery at the prehilar bifurcation. A full color version of this figure is available at the Hypertension Research journal online.

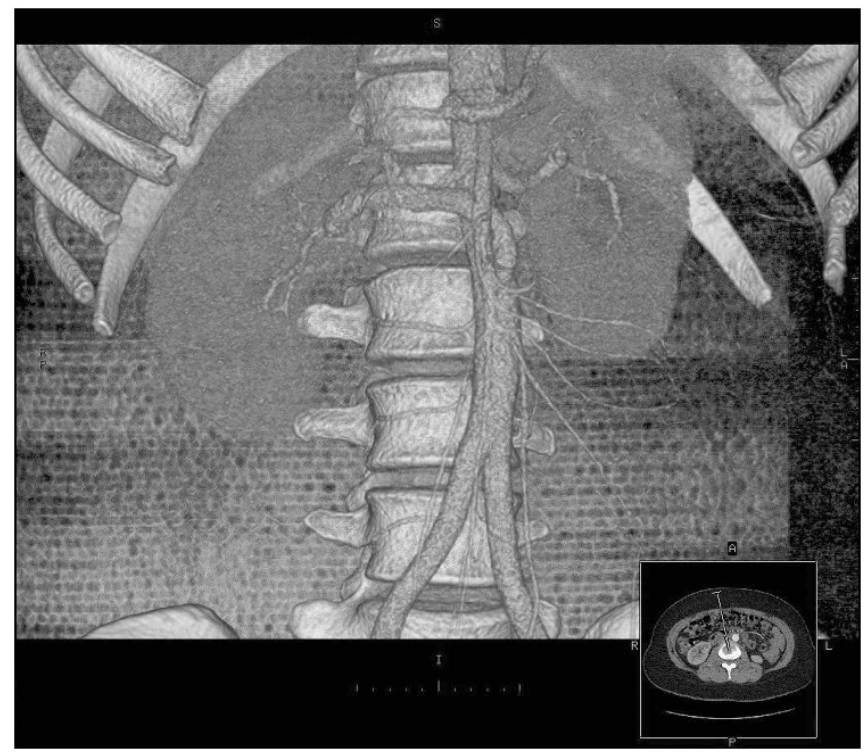

Figure 3 Abdominal CT scan 2 years after surgical revascularization showing the venous bypass. A full color version of this figure is available at the Hypertension Research journal online.

medications, reduction of renal size or reduction of renal function.

PTA is the recommended first-step therapy, as it is very effective with no need for stent implantation in most circumstances. Stenting is considered for treating dissection and if the gradient cannot be obliterated with angioplasty alone. ${ }^{2}$ According to current guidelines, ${ }^{3}$ surgical revascularization should be performed to treat aneurysms in patients in whom endovascular therapy is not an option or after PTA failure, despite the observations that it controls hypertension better than endovascular therapy, ${ }^{4}$ and it provides the most durable long-term results. ${ }^{5}$ Furthermore, vascular surgical reconstruction is indicated for patients with FMD renal artery stenosis exhibiting complex disease that extends into the segmental arteries and those having macro aneurysms. ${ }^{3}$

In this patient, we offered surgical revascularization at the first attempt because to our judgment the stenosis was close to artery bifurcation and any artery dissection may have jeopardised renal perfusion, thus requiring stent implantation in a young patient or in the case of failure, emergency surgical treatment. As there is no consideration of the site of stenosis in the current guidelines, we suggest that the site of stenosis is included among the vascular characteristics that guide treatment choices for FMD.

Martino F Pengo ${ }^{1}$, Roberto Ragazzi ${ }^{2}$, Claudio Fittà ${ }^{2}$, Franco Grego ${ }^{3}$ and Andrea Semplicini ${ }^{1,4}$

${ }^{1}$ Department of Medicine, University of Padua, Padua, Italy; ${ }^{2}$ Unit of Radiology, USL12 Veneziana, Venice, Italy; ${ }^{3}$ Clinic of Vascular and Endovascular Surgery, University of Padua, Padua, Italy and ${ }^{4}$ Unit of Internal Medicine 1, USL12 Veneziana, Venice, Italy

E-mail: andrea.semplicini@unipd.itor andrea.semplicini@ulss12.ve.it

1 Sabharwal R, Vladica P, Law WP. Multidetector spiral CT renal angiography in the diagnosis of renal artery fibromuscular dysplasia. Eur J Radiol 2007; 61: 520-527

2 Weiner RB, Kiernan TJ, Yan BP, Rosenfield K, Jaff MR Images in cardiovascular medicine. Adventitial fibromuscular dysplasia of the renal artery: management challenges of nonatherosclerotic renal artery stenosis. Circulation 2009; 120: e157-e158.

3 Hirsch AT, Haskal ZJ, Hertzer NR, Bakal CW, Creager MA, Halperin JL, Hiratzka LF, Murphy WR, Olin JW, Puschett JB, Rosenfield KA, Sacks D, Stanley JC, Taylor Jr LM, White CJ, White J, White RA, Antman EM, Smith Jr SC, Adams CD, Anderson JL, Faxon DP, Fuster V, Gibbons RJ, Halperin JL, Hiratzka LF, Hunt SA, Jacobs AK, Nishimura R, Ornato JP, Page RL, Riegel B; American Association for Vascular Surgery; Society for Vascular Surgery; Society for Cardiovascular Angiography and Interventions; Society for Vascular Medicine and Biology; Society of Interventional Radiology; ACC/AHA Task Force on Practice Guidelines; American Association of Cardiovascular and Pulmonary Rehabilitation; National Heart, Lung, and Blood Institute; Society for Vascular Nursing. TransAtlantic Inter-Society Consensus; Vascular Disease Foundation. ACC/AHA 2005 guidelines for the management of patients with peripheral arterial disease (lower extremity, renal, mesenteric, and abdominal aortic). J Am Coll Cardiol 2006; 47: 1239-1312.

4 Trinquart L, Mounier-Vehier C, Sapoval M, Gagnon N, Plouin PF. Efficacy of revascularization for renal artery stenosis caused by fibromuscular dysplasia: a systematic review and meta-analysis. Hypertension 2010; 56 525-532.

5 Ham SW. Late outcomes of endovascular and open revascularization for nonatherosclerotic renal artery disease. Arch Surg 2010; 145: 832-839. 